\section{JTI}

JOURNAL OF

TRAUMA AND INJURY

Received: August 4, 2020

Revised: September 29, 2020

Accepted: October 12, 2020

\section{Correspondence to}

Hohyun Kim, M.D.

Department of Trauma and Surgical Critical Care, Pusan National University Hospital, 179 Gudeok-ro, Seo-gu, Busan 49241, Korea

Tel: + 82-51-240-7369

Fax: +82-51-240-7719

E-mail: traumagskhh@naver.com

\title{
Incidence and Clinical Features of Urethral Injuries with Pelvic Fractures in Males: A 6-Year Retrospective Cohort Study at a Single Institution in South Korea
}

\author{
Hyun Woo Sun, M.D. ${ }^{1}$, Hohyun Kim, M.D. ${ }^{2,3}$, Chang Ho Jeon, M.D. ${ }^{4}$, \\ Jae Hoon Jang, M.D. ${ }^{3,5}$, Gil Hwan Kim, M.D. ${ }^{2,3}$, Chan Ik Park, M.D. ${ }^{2,3}$, \\ Sung Jin Park, M.D. ${ }^{2,3}$, Jae Hun Kim, M.D. ${ }^{2,3}$, Seok Ran Yeom, M.D., \\ ${ }^{1}$ Department of Intensive Care Medicine, Dong-A University Hospital, Busan, Korea \\ ${ }^{2}$ Department of Trauma and Surgical Critical Care, Pusan National University Hospital, \\ Busan, Korea \\ ${ }^{3}$ Biomedical Research Institute, Pusan National University Hospital, Busan, Korea \\ ${ }^{4}$ Department of Diagnostic Radiology, The Catholic University of Korea Eunpyeong St. \\ Mary's Hospital, Seoul, Korea \\ ${ }^{5}$ Department of Orthopedic Surgery, Pusan National University Hospital, Busan, Korea \\ ${ }^{6}$ Department of Emergency Medicine, Pusan National University Hospital, Busan, Korea
}

Purpose: Severe pelvic fractures are associated with genitourinary injuries, but the relationship between pelvic trauma and concomitant urethral injuries has yet to be elucidated. This study evaluated the incidence, mechanism, site, and extent of urethral injuries in male patients with pelvic fractures.

Methods: A retrospective cohort study was performed involving patients with urethral injuries accompanying pelvic fractures who visited Pusan National University Hospital from January 1, 2014 to December 31, 2019. Demographics, mechanisms of injury, clinical features of the urethral injuries, concomitant bladder injuries, methods of management, and the configuration of the pelvic fractures were analyzed.

Results: The final study population included 24 patients. The overall incidence of urethral injury with pelvic fracture was $2.6 \%$, with the most common mechanism of urethral injury being traffic accidents (62.5\%). Complete urethral disruption (16/24, 66.7\%) was more common than partial urethral injuries $(8 / 24,33.3 \%)$, and unstable pelvic fractures were the most common type of pelvic fracture observed (70.8\%). There was no definitive relationship between the extent of urethral injury and pelvic ring stability. Conclusions: The present study provides a 6-year retrospective review characterizing the incidence, mechanism, and clinical features of urethral injury-associated pelvic fractures. This study suggests that the possibility of urethral injury must be considered, especially in unstable pelvic fracture patients, and that treatment should be chosen based on the clinical findings.

Keywords: Urethra; Incidence; Pelvic bones; Multiple trauma 


\section{INTRODUCTION}

Pelvic fractures are often caused by high-energy events, with the most common causes including motor vehicle collisions, motorcycle collisions, auto-pedestrian collisions, and falls from heights [1-4]. Furthermore, the urethra in males is more vulnerable to injuries due to anatomical differences between males and females. Complete disruption of the male urethra occurs in two-thirds of injuries, with partial injuries accounting for the remaining third [5].

Although previous studies have suggested that urethral injuries occur in up to $10 \%$ of male patients with pelvic fractures, the incidence and etiology of traumatic urethral injuries remain to be fully elucidated [6,7]. Previous studies have been based on relatively small patient populations, and none have thoroughly described differences in presentation and outcomes according to the site and extent of urethral injury-associated pelvic fractures [8].

This study reviewed data over a 6-year period from a single institution from patients with urethral injuries and pelvic fractures. The aim of this study was to report the incidence, mechanism, site, and extent of urethral injuries with pelvic fractures in males.

\section{METHODS}

\section{Study setting}

A retrospective cohort study was performed at a regional trauma center between January 1, 2014 and December 31, 2019. Patients were identified using the hospital inpatient enquiry system. We retrospectively reviewed data from these medical records and included a total of 12,891 patients with traumatic injuries who were admitted to Regional Trauma Center, Pusan National University Hospital.

All patients with pelvic fractures were included. The exclusion criteria were patients who were $<16$ years old, female, declared dead on arrival, or discharged/transferred from the emergency room, as well as those with unclear medical records or no urethral injury. The final study population consisted of 24 patients with urethral injuries (Fig. 1). The patients' demographic characteristics, mechanisms of injury, and details of injuries were recorded. Available data included age, sex, mechanisms of injury, Injury Severity Score (ISS), clinical features of the urethral injury, methods of management, configuration of the pelvic fracture, the Abbreviated Injury Scale score for pelvic ring fracture, the length of hospital stay, the intensive care unit (ICU) length of stay (LOS), and survival status.

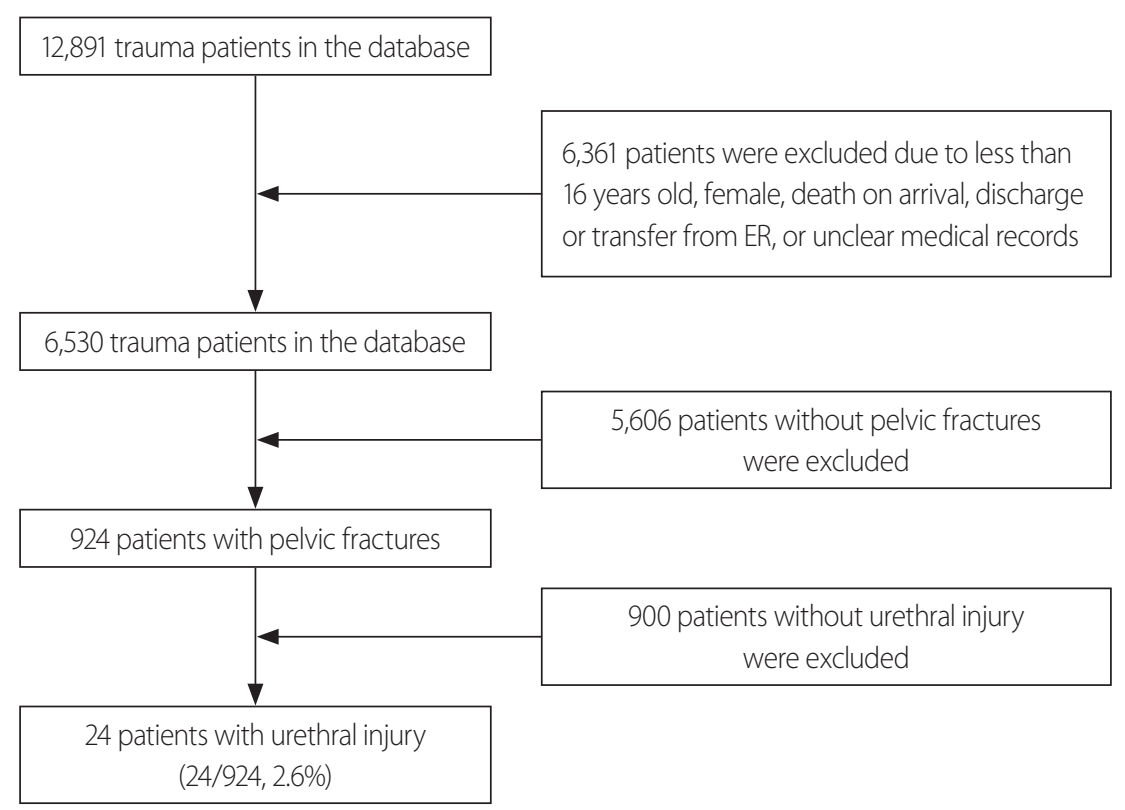

Fig. 1. Study flowchart. ER: emergency room. 


\section{Injuries recorded}

\section{Urethral injury}

The demographic characteristics of patients with urethral injuries and pelvic fractures were recorded. The patients were subdivided into two broad categories based on whether the urethral injury was anterior or posterior urethral. The anterior urethra included the bulbar urethra, the pendulous urethra, and the fossa navicularis, while the posterior urethra included the bladder neck, the prostatic urethra, and the membranous urethra. The patients were also subdivided into two broad categories according to whether the extent of urethral injury involved partial or complete disruption. Partial disruption was defined as extravasation of contrast at the injury site with contrast visualized in the proximal urethra or bladder [9]. Complete disruption was defined as a visible discontinuity of the urethra or extravasation of contrast at the injury site without visualization of the proximal urethra, anterior urethra, or the bladder [9]. Follow-up was conducted every 3 months for 6 months after injury, and then every 6 months for the next 2 years at the urology outpatient clinic.

\section{Orthopedic injuries}

A pelvic fracture was defined as a pelvic ring fracture and/ or acetabular fracture. Details of the pelvic ring fractures, including the fracture type according to the Young and Burgess [10,11] and Tile [12] classifications of pelvic fractures, were recorded. The Young and Burgess system classifies pelvic fractures into lateral compression (LC) types $1-3$, anterior-posterior compression (APC) types $1-3$, the vertical shear (VS) type, and the combined (CM) type. In contrast, the Tile system classifies fractures into types A, B, and C. Pelvic fractures were subdivided into three broad categories: stable pelvic fractures, partially unstable pelvic fractures, and unstable pelvic fractures. Stable pelvic fractures were defined as APC 1 [13], partially unstable pelvic fractures were defined as LC 1 or APC 2 [13] and unstable pelvic fractures were defined as LC 2, LC 3, APC 3, VS, or CM [13].

\section{Outcome measures}

The primary objective was to report the incidence, mech- anism, site, and extent of urethral injuries in patients with pelvic fractures. The secondary objective was to evaluate the relationship between the extent of urethral injury and the level of pelvic ring stability.

\section{Statistical analyses}

Summary statistics are reported as the median and interquartile range (IQR) as appropriate. Categorical variables are expressed as numbers and percentages. The Fisher exact test was used to compare the frequencies of categorical variables between groups. A $p$-value $<0.05$ was considered to indicate statistical significance. Stata version 14.2 (Stata Corp., College Station, TX, USA) was used to analyze the data.

\section{RESULTS}

\section{Patients' characteristics and clinical features}

The demographic characteristics of the patients with urethral injuries are summarized in detail in Table 1. The overall incidence of urethral injuries with pelvic fractures was $2.6 \%$ (24 of 924) (Fig. 1). The median age of the patients was 59 years (IQR 40-67 years) with the most common mechanism being traffic accidents (62.5\%). Out of the 24 urethral injuries, 20 were classified as posterior $(85.3 \%)$, while the other four were classified as anterior (16.7\%). There were 16 complete urethral injuries (66.7\%) and eight partial disruptions of the urethra (33.3\%). The median ISS was 28 (IQR 20-34) and concomitant bladder injuries occurred in five out of 24 patients (20.8\%).

Formal retrograde urethrography was performed in $70.8 \%$ of urethra trauma cases during the initial hospitalization (partial injuries: three cases; complete injuries: 12 cases). Two cases $(8.3 \%)$ of urethral injury were diagnosed via computed tomography $(\mathrm{CT})$ findings (partial injuries: one case; complete injuries: one case). The remainder (20.8\%) were diagnosed based on intraoperative findings (partial injuries: two cases; complete injuries: three cases).

Eighteen patients $(75.0 \%)$ were treated with non-operative management, while the remainder were treated with operative management ( $n=6,25.0 \%$ ). Non-operative management consisted of interventional urethral realignment or insertion of a Foley catheter. 
Table 1. Clinical features of patients with urethral injuries associated with pelvic fractures

\begin{tabular}{|c|c|}
\hline Characteristics & Total $(n=24)$ \\
\hline Age (years) & $59(40-67)$ \\
\hline \multicolumn{2}{|l|}{ Injury mechanism } \\
\hline Occupant TA & $4(16.7)$ \\
\hline Motorcycle TA & $3(12.5)$ \\
\hline Pedestrian TA & $8(33.3)$ \\
\hline Fall & $1(4.2)$ \\
\hline Entrapment & $7(29.2)$ \\
\hline Others & $1(4.2)$ \\
\hline \multicolumn{2}{|l|}{ Injury site } \\
\hline Anterior urethra & $4(16.7)$ \\
\hline Posterior urethra & $20(83.3)$ \\
\hline \multicolumn{2}{|l|}{ Extent of injury } \\
\hline Complete disruption & $16(66.7)$ \\
\hline Partial injury & $8(33.3)$ \\
\hline ISS & $28(20-34)$ \\
\hline Concomitant bladder injury & $5(20.8)$ \\
\hline \multicolumn{2}{|l|}{ Diagnosis } \\
\hline $\mathrm{CT}$ & $2(8.3)$ \\
\hline Urethrography & $17(70.8)$ \\
\hline Intraoperative & $5(20.8)$ \\
\hline \multicolumn{2}{|l|}{ Management } \\
\hline Operative management & $6(25.0)$ \\
\hline Non-operative management ${ }^{\mathrm{a}}$ & $18(75.0)$ \\
\hline \multicolumn{2}{|l|}{ Outcome } \\
\hline Hospital stay (days) & $31(20-49)$ \\
\hline ICU stay (days) & $2(1-5)$ \\
\hline Mortality & 0 \\
\hline Delayed complication of urethral injury & $15(62.5)$ \\
\hline Urethral stenosis & $13(54.2)$ \\
\hline Urinary incontinence & $2(8.3)$ \\
\hline Erectile dysfunction & $2(8.3)$ \\
\hline
\end{tabular}

Values are presented as median (interquartile range) or number (\%).

TA: traffic accident, ISS: Injury Severity Score, CT: computed tomography, ICU: intensive care unit.

${ }^{a}$ Non-operative management was defined as interventional urethral realignment or insertion of a Foley catheter.

The median duration of hospital stay and ICU LOS were 31 days (IQR 20-49 days) and 2 days (IQR 1-5
Table 2. Configurations of pelvic fractures in patients with urethral injuries

\begin{tabular}{|lc|}
\hline Characteristics & Total $(\mathbf{n}=\mathbf{2 4})$ \\
AIS for pelvic ring & \\
$<3$ & $3(12.5)$ \\
$\geq 3$ & $21(87.5)$ \\
Young-Burgess classification & \\
APC & $3(12.5)$ \\
LC & $18(75.0)$ \\
VS or CM & $3(12.5)$ \\
Tile classification & \\
A & $1(4.2)$ \\
B & $19(79.1)$ \\
C & $4(16.7)$ \\
Stability of fracture & \\
Stable & $1(4.2)$ \\
Partially unstable & \\
Unstable $^{c}$ & $6(25.0)$ \\
\hline
\end{tabular}

Values are presented as number (\%).

AIS: Abbreviated Injury Scale, APC: anterior-posterior compression, LC: lateral compression, VS: vertical shear, CM: combined.

astable pelvic fracture was defined as APC 1 type.

${ }^{b}$ Partially unstable pelvic fractures were defined as LC 1 or APC 2 types.

'Unstable pelvic fractures were defined as LC 2, LC 3, APC 3, VS, or CM types.

days), respectively. No deaths were reported during the initial hospitalization. Delayed complications occurred in 15 cases (62.5\%). Urethral stenosis occurred in 13 patients $(54.2 \%)$, and urinary incontinence and erectile dysfunction occurred in one patient each (8.3\%). Delayed urethral complications were more common in patients with complete urethral injuries than in patients with partial injuries, but there was no statistically significant difference between the two groups ( $62.5 \%$ vs. $50.0 \%, p=0.673$ ).

\section{Configuration of pelvic fractures in patients with ure- thral injuries}

The configurations of pelvic fractures in patients with urethral injuries are shown in Table 2. LC was the most common fracture type according to the Young and Burgess system (18 of $24 ; 75.0 \%$ ), whereas type B was the most common fracture type according to the Tile system (19 of 24; 79.1\%). Unstable pelvic fractures (LC 2, LC 3, 
Table 3. Relationship between the extent of urethral injuries and the configuration of pelvic fractures

\begin{tabular}{|c|c|c|c|}
\hline Characteristics & Partial disruption $(\mathrm{n}=8)$ & Complete disruption $(n=16)$ & $p$-value \\
\hline AlS for pelvic ring & & & 1.000 \\
\hline$<3$ & $1(12.5)$ & $2(12.5)$ & \\
\hline$\geq 3$ & $7(87.5)$ & $14(87.5)$ & \\
\hline Young-Burgess classification & & & 0.055 \\
\hline APC & $3(37.5)$ & 0 & \\
\hline LC & $4(50.0)$ & $14(87.5)$ & \\
\hline VS or CM & $1(12.5)$ & $2(12.5)$ & \\
\hline Tile classification & & & 0.505 \\
\hline A & $1(12.5)$ & 0 & \\
\hline B & $6(75.0)$ & $13(81.2)$ & \\
\hline C & $1(12.5)$ & $3(18.8)$ & \\
\hline Stability of fracture & & & 0.193 \\
\hline Stable ${ }^{a}$ & $1(12.5)$ & 0 & \\
\hline Partially unstable $e^{b}$ & $3(37.5)$ & $3(18.7)$ & \\
\hline Unstable & $4(50.0)$ & $13(81.3)$ & \\
\hline
\end{tabular}

Values are presented as number (\%).

AIS: Abbreviated Injury Scale, APC: anterior-posterior compression, LC: lateral compression, VS: vertical shear, CM: combined.

aStable pelvic fracture was defined as APC 1 type.

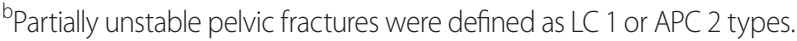

'Unstable pelvic fractures were defined as LC 2, LC 3, APC 3, VS, or CM types.

APC 3, VS, or CM) occurred in 70.8\% ( $\mathrm{n}=17)$ of patients, and partially unstable pelvic fractures (LC 1 or APC 2) occurred in $25.0 \%(\mathrm{n}=6)$.

\section{Relationship between the extent of urethral injuries and the configuration of pelvic fractures}

Table 3 demonstrates the relationship between the extent of urethral injuries (partial vs. complete) and the configuration of pelvic fractures. The risk of complete disruption of the urethra was higher in patients with unstable pelvic fractures than in those with stable or partially unstable fractures $(81.3 \%$ vs. $0 \%$ vs. $18.7 \%)$. However, this result was not statistically significant $(p=0.193)$. There was no definitive relationship between the extent of urethral injury and the specific type of pelvic fracture.

\section{DISCUSSION}

This study was the first to evaluate the incidence, mech- anism, site, and extent of urethral injuries in a large contemporary cohort in South Korea. We found that the overall incidence of urethral injuries in males from blunt pelvic trauma was $2.6 \%$ of all pelvic fractures. No significant relationship was found between the extent of urethral injury and the severity of pelvic ring stability. However, we believe that this is likely due to the rarity of urethral injuries and the lack of sufficient statistical power to detect a significant difference.

Although the urethra is deeply located in the pelvic cavity, its close proximity to the pelvic bone may lead to injury during severe pelvic trauma. Urethral injuries usually occur in the setting of significant pelvic fractures, often from motor vehicle collisions or crush injuries [4]. Pelvic fracture-associated urethral injuries occur in $1.6 \%$ to $25 \%$ of pelvic fractures, corresponding to $0.32-5 / 100,000$ males [14-17]. Combined urethral and bladder injuries occur in $1-33 \%$ of patients with pelvic fractures [14,1619]. These wide variations may be due to variations in the use of diagnostic tools such as urethrography or comput- 
ed tomography. Delayed complications such as urethral stricture, erectile dysfunction, and urinary incontinence may occur in up to $90 \%$ of patients with urethral injuries [14]. Our study found comparable rates of the incidence of urethral injury associated with pelvic fractures in males $(2.6 \%)$, concomitant bladder injuries $(20.8 \%)$, and delayed complications (62.5\%).

Our study indicated that the risk of developing a complete disruption of the urethra was higher in patients with unstable pelvic fractures than in those with partially unstable or stable fractures. Urethral injuries in the setting of a pelvic fracture are associated with mechanisms that rupture the puboprostatic ligament with or without shearing forces to the perineal membrane [14]. Pelvic fractures can be associated with either partial or complete urethral disruption. However, the latter is more common [9]. Complete urethral disruption occurs when a significant pelvic fracture causes upward displacement of the bladder and prostate. Avulsion of the puboprostatic ligament is followed by stretching of the membranous urethra, resulting in complete disruption at the anatomic weak point, the bulbomembranous junction [4]. We think that this mechanism may explain why complete urethral disruption was common in patients with unstable pelvic fractures.

From a clinical perspective, the characteristics of a pelvic fracture are useful in determining the likelihood of urethral injury [13]. Using a mechanistic understanding of urethral injury, previous investigators have attempted to predict injuries based on particular fracture configurations [7,13,20,21]. Battaloglu et al. [13] reported that urethral injuries were associated with unstable pelvic fractures, but not with a specific pelvic fracture type. We found that posterior and complete urethral injury rates tended to rise as stability declined; however, this relationship was not statistically significant. We suggest that more work is needed to evaluate the relationship between urethral injuries and pelvic ring stability.

There are several limitations to this study. First, the study was confined to patients at a single center, and our study population may have been specific to a certain region; additionally, this study only analyzed the population of patients who presented to a regional trauma center. As such, the severity and frequency of injuries seen at Pusan National University Hospital likely differ significantly from those seen at a community emergency department. Second, because this was a non-randomized and retrospective analysis with a limited number of cases, the results are not conclusive and lacked sufficient statistical power to detect a significant differences. Therefore, additional prospective studies involving larger sample sizes will be necessary to support our findings.

\section{CONCLUSION}

This 6-year single-institution cohort study involved patients with urethral injuries with pelvic fractures. Urethral injuries occurred in $2.6 \%$ of all pelvic fracture patients. Complete disruption was more common than partial urethral injuries, and unstable pelvic fracture was the most common fracture type. In addition, the risk of complete disruption was found to rise as stability declined. This study emphasizes the importance of maintaining a high index of clinical suspicion in patients who present with urethral injuries, especially in those with unstable pelvic fractures. Further research involving a larger number of cases is needed in order to clarify the relationship between urethral injuries and the configuration of pelvic fractures.

\section{REFERENCES}

1. Balogh Z, King KL, Mackay P, McDougall D, Mackenzie S, Evans JA, et al. The epidemiology of pelvic ring fractures: a population-based study. J Trauma 2007;63:1066-73; discussion 10723.

2. Durkin A, Sagi HC, Durham R, Flint L. Contemporary management of pelvic fractures. Am J Surg 2006;192:211-23.

3. Grotz MR, Allami MK, Harwood P, Pape HC, Krettek C, Giannoudis PV. Open pelvic fractures: epidemiology, current concepts of management and outcome. Injury 2005;36:1-13.

4. Koraitim MM. Pelvic fracture urethral injuries: the unresolved controversy. J Urol 1999;161:1433-41.

5. Webster GD, Mathes GL, Selli C. Prostatomembranous urethral injuries: a review of the literature and a rational approach to their management. J Urol 1983;130:898-902.

6. Demetriades D, Karaiskakis M, Toutouzas K, Alo K, Velmahos G, Chan L. Pelvic fractures: epidemiology and predictors of 
associated abdominal injuries and outcomes. J Am Coll Surg 2002;195:1-10.

7. Basta AM, Blackmore CC, Wessells H. Predicting urethral injury from pelvic fracture patterns in male patients with blunt trauma. J Urol 2007;177:571-5.

8. Dixon AN, Webb JC, Wenzel JL, Wolf JS Jr, Osterberg EC. Current management of pelvic fracture urethral injuries: to realign or not? Transl Androl Urol 2018;7:593-602.

9. Martínez-Piñeiro L, Djakovic N, Plas E, Mor Y, Santucci RA, Serafetinidis E, et al. EAU guidelines on urethral trauma. Eur Urol 2010;57:791-803.

10. Burgess AR, Eastridge BJ, Young JW, Ellison TS, Ellison PS Jr, Poka A, et al. Pelvic ring disruptions: effective classification system and treatment protocols. J Trauma 1990;30:848-56.

11. Alton TB, Gee AO. Classifications in brief: young and burgess classification of pelvic ring injuries. Clin Orthop Relat Res 2014;472:2338-42.

12. Tile M. Pelvic ring fractures: should they be fixed? J Bone Joint Surg Br 1988;70:1-12.

13. Battaloglu E, Figuero M, Moran C, Lecky F, Porter K. Urethral injury in major trauma. Injury 2019;50:1053-7.

14. Barratt RC, Bernard J, Mundy AR, Greenwell TJ. Pelvic fracture urethral injury in males-mechanisms of injury, management options and outcomes. Transl Androl Urol 2018;7(Suppl 1):S29-62.
15. Lückhoff C, Mitra B, Cameron PA, Fitzgerald M, Royce P. The diagnosis of acute urethral trauma. Injury 2011;42:913-6.

16. Sandler CM, Harris JH Jr, Corriere JN Jr, Toombs BD. Posterior urethral injuries after pelvic fracture. AJR Am J Roentgenol 1981;137:1233-7.

17. Palmer JK, Benson GS, Corriere JN Jr. Diagnosis and initial management of urological injuries associated with 200 consecutive pelvic fractures. J Urol 1983;130:712-4.

18. Bjurlin MA, Fantus RJ, Mellett MM, Goble SM. Genitourinary injuries in pelvic fracture morbidity and mortality using the National Trauma Data Bank. J Trauma 2009;67:1033-9.

19. Eidelman E, Stormont I, Churukanti G, Shreck E, Belay R, Capodice $S$, et al. Injury severity score associated with concurrent bladder injury in patients with blunt urethral injury. World J Urol 2019;37:983-8.

20. Service CA, Moses RA, Majercik SD, Hotaling JM, Keihani S, Rothberg D, et al. Urethral trauma following pelvic fracture from horseback saddle horn injury versus other mechanisms of pelvic trauma. Urology 2019;124:260-3.

21. Aihara R, Blansfield JS, Millham FH, LaMorte WW, Hirsch EF. Fracture locations influence the likelihood of rectal and lower urinary tract injuries in patients sustaining pelvic fractures. J Trauma 2002;52:205-8; discussion 208-9. 